\title{
Episclerite tuberculosa em paciente com doença autoimune: um relato de caso
}

\author{
Tuberculous episcleritis in a patient with autoimmune disease: a case report
}

Epiescleritis tuberculosa en un paciente con enfermedad autoinmune: un reporte de caso

Murilo Labre Tavares ${ }^{1 *}$, Isabela Cordeiro de Sousa ${ }^{1}$, Ronaldo Luís Oliveira Delgado ${ }^{1}$, Thácila Fernandes de Sousa ${ }^{1}$, Laura Barcelos Azzam¹, Nubia Cristina de Freitas Maia ${ }^{1}$.

\section{RESUMO}

Objetivo: Relatar um caso sobre episclerite tuberculosa e os desafios clínicos para realizar o diagnóstico. Detalhamento do caso: Paciente masculino, 20 anos, natural e residente do Estado do Tocantins e portador de espondilite psoriásica. Procurou atendimento oftalmológico queixando-se de irritação em olho esquerdo há 20 dias com acumulo de secreção matinal. Realizada biomicroscopia ocular que revelou quadro de conjuntivite crônica ativa inespecífica, não respondendo ao tratamento inicial. Realizado biópsia da lesão com pesquisa para fungos, com presença destes. Prescrito tratamento para infecção fúngica, que apresentou melhora com posterior piora clínica. Realizada nova biópsia, que revelou inflamação, sem hifas, sendo cogitada a hipótese de tuberculose ocular. Realizado PPD que resultou reação de $21 \mathrm{~mm}$, sendo instituído tratamento específico com resolução do quadro, fechando o diagnóstico de epiesclerite tuberculosa. Considerações finais: A tuberculose apresenta alta prevalência no Brasil e pode ter manifestações pulmonares e extrapulmonares, como a tuberculose ocular. Como demonstrado, há dificuldade no diagnóstico desta patologia e por isso este relato pretende chamar a atenção dos profissionais da saúde para o diagnóstico desta.

Palavras chave: Tuberculose, Episclerite, Oftalmologia.

\begin{abstract}
Objective: Report a case report of tuberculous episcleritis and the clinical challenges to make the diagnosis. Case details: Male patient, 20 years old, born and resident of the State of Tocantins and with psoriatic spondylitis. He sought eye care complaining of irritation in the left eye for 20 days with an accumulation of morning secretion. Ocular biomicroscopy was performed and revealed a clinical picture of nonspecific active chronic conjunctivitis, not responding to the initial treatment. Biopsy of the lesion was performed with research for fungi, with the presence of these. Treatment for fungal infection was prescribed, which improved with subsequent clinical worsening. A new biopsy was performed, which revealed inflammation, without hyphae, and the hypothesis of ocular tuberculosis was considered. PPD was performed, which resulted in a $21 \mathrm{~mm}$ reaction, and specific treatment was instituted with resolution of the condition, concluding the diagnosis of tuberculous episcleritis. Final considerations: Tuberculosis is highly prevalent in Brazil and may have pulmonary and extrapulmonary manifestations, such as ocular tuberculosis. As shown, there is difficulty in diagnosing this pathology and, therefore, this report intends to draw the attention of health professionals to its diagnosis.
\end{abstract}

Keywords: Tuberculosis, Episcleritis, Ophthalmology.

${ }^{1}$ Universidade Federal do Tocantins, Palmas - TO. *E-mail: mlabretavares@gmail.com

SUBMETIDO EM: 6/2021

ACEITO EM: 7/2021

PUBLICADO EM: $8 / 2021$ 


\section{RESUMEN}

Objetivo: Reportar un caso clínico sobre la epiescleritis tuberculosa y los desafíos clínicos para el diagnóstico. Detalles del caso: Paciente de sexo masculino, 20 años, nacido y residente del estado de Tocantins y con espondilitis psoriásica. Buscó atención oftalmológica quejándose de irritación en el ojo izquierdo durante 20 días con acumulación de secreciones matutinas. Se realizó biomicroscopía ocular que reveló un cuadro clínico de conjuntivitis crónica activa inespecífica, que no respondía al tratamiento inicial. Se realizó biopsia de la lesión con investigación de hongos, con presencia de estos. Se prescribió tratamiento para la infección por hongos, que mejoró con el posterior empeoramiento clínico. Se realizó una nueva biopsia, que reveló inflamación, sin hifas, y se consideró la hipótesis de tuberculosis ocular. Se realizó PPD, que resultó en una reacción de $21 \mathrm{~mm}$, y se instauró tratamiento específico con resolución del cuadro, concluyendo el diagnóstico de epiescleritis tuberculosa. Consideraciones finales: La tuberculosis es muy prevalente en Brasil y puede tener manifestaciones pulmonares y extrapulmonares, como la tuberculosis ocular. Como se muestra, existe dificultad en el diagnóstico de esta patología y, por lo tanto, este informe pretende llamar la atención de los profesionales de la salud sobre su diagnóstico.

Palabras clave: Tuberculosis, Epiescleritis, Oftalmología.

\section{INTRODUÇÃO}

Define-se tuberculose ocular como uma patologia infecciosa causada pelo Mycobacterium tuberculosis dentro do globo ocular, ou em sua superfície. Na tuberculose sistêmica (TB), a manifestação ocular pode ocorrer em 1-2\% dos casos (CÂMARA SDN, et al., 2019). A tuberculose ocular é caracterizada como primária, uma vez que o olho é o foco inicial da infecção pela bactéria; e coloca-se como infecção secundária, quando o acometimento ocular ocorre por via hematogênica, que pode levar ao comprometimento de todas as estruturas que compõem o olho e seus anexos, seja por ação direta do agente patogênico ou por um processo de reação imunológica de hipersensibilidade do tipo IV (KOLLER K, et al., 2019; SHAKARCHI FI, 2015).

E entre essas estruturas que compõem a anatomia ocular, é possível observar especificamente a episclera conjuntival, que compõe a parte superficial do olho e é constituída por tecido conjuntivo vascular denso originário do estroma escleral de superfície em conjunto com o material da cápsula de Tenon que a sobrepõe, sendo a camada superficial da esclera. As funções desempenhadas pela episclera são de dar suporte nutricional, revestimento e proteção através de uma resposta inflamatória para a estrutura da esclera conjuntival. As patologias mais frequentes da esclera e da episclera são aquelas do grupo das inflamatórias, ocorrendo ainda enfermidades metabólicas, degenerativas, neoplásicas e por mecanismo de trauma (MARTINEZ AAG, et al., 2013).

Dentre as patologias inflamatórias que afetam a episclera e a esclera, a subclasse das infecciosas são bastante infrequentes, representando apenas $7 \%$ das patologias de cunho inflamatório; os relatos existentes são decorrentes de infecção por hanseníase, tuberculose, brucelose, Herpes zoster, sífilis e doença de Lyme (FÉNOLLAND JR, et al., 2016; LOUREIRO M, et al., 2016; DOSHI RR, et al., 2013; REDDY JC, et al., 2015; FERREIRA REBELO PB, et al., 2019).

O quadro clínico é de dor ocular moderada à severa, edema palpebral, proptose, hiperemia ocular, lacrimejamento, fotofobia, podendo ou não apresentar baixa acuidade visual (KOLLER K, et al., 2019; GUPTA V, et al., 2015; AMARAL GR, et al., 2020). O diagnóstico diferencial com as demais formas de episclerites inflamatórias é dificultoso, sendo suspeitado quando não há melhora do quadro inflamatório com o tratamento anti-inflamatório e associado ao diagnóstico faz-se testes sorológicos e reacionais para um determinado agente etiológico (AMARAL GR, et al., 2020).

O diagnóstico específico de tuberculose ocular é penoso pela dificuldade de se isolar o agente Mycobacterium tuberculosis e em biópsias e raspados da lesão, sendo esse realizado, então, de forma presuntiva na grande maioria dos casos, considerando-se para tanto a epidemiologia positiva, a clínica sistêmica e ocular e a resposta imunológica do paciente (REBELLO AC, et al., 2015; AMARAL GR, et al., 
2020). Vale-se também de exames complementares para o diagnóstico, como radiografias de tórax e estudo de teste tuberculínico (PPD); assim como a hiporresponsividade ao tratamento com esteroides e resposta positiva à quimioterapia antituberculosa (AMARAL GR, et al., 2020; CAMPOS WR, et al., 2021).

Uma vez constatada as altas incidência e prevalência da TB no mundo, e em especial na população brasileira, a ampliação dos conhecimentos a respeitos das diversas formas de manifestação clínica, além dos quadros pulmonares, é de grande importância; assim como as metodologias diagnósticas praticadas e os protocolos de tratamento para essa infecção (BRASIL, 2019; LAIOS K, et al., 2017).

O presente relato de caso objetivou apresentar: os aspectos clínicos que podem ser manifestados na episclerite tuberculosa; os desafios e dificuldades da clínica para o estabelecimento do diagnóstico; discutir o estabelecimento de uma conduta terapêutica uma vez que estabelecida a hipótese de tuberculose em uma estrutura ocular; e o seguimento do paciente até a remissão da lesão.

\section{DETALHAMENTO DO CASO}

M.L.T, sexo masculino, 20 anos de idade, natural e residente do estado do Tocantins. Portador de espondilite psoriásica com diagnóstico aos 19 anos, após investigação de dor importante em região pélvica unilateral. Ressonância Magnética evidenciando inflamação crônica de articulação entre ílio e ísquio em lado direito em novembro de 2016. Faz tratamento com Naproxeno $500 \mathrm{mg}$ duas vezes ao dia com bom controle da doença.

Procurou atendimento oftalmológico queixando-se de irritação no Olho Esquerdo (OE) há 20 dias, associada a acúmulo de secreção ocular pela manhã. Ao exame de Biomicroscopia Ocular (BIO) em OE, foi observada área hiperemiada e bem delimitada na conjuntiva superior, com lesões em área central corando por fluoresceína, pequenos pontos de infiltrado corneano subepiteliais na periferia corneana superior e inferior, alterações conjuntivais semelhantes e mais leves em conjuntiva inferior. Prescrito gatifloxacino + acetato de prednisolona suspensão oftálmica 6 vezes ao dia.

Diante da pouca melhora clínica após duas semanas do uso da medicação, foi realizada uma biópsia da lesão, que revelou intenso infiltrado inflamatório misto, rico em plasmócitos, linfócitos, eosinófilos e neutrófilos, consistente com conjuntivite crônica ativa inespecífica. A pesquisa de Bacilos Álcool-Ácido Resistentes (BAAR) foi negativa, entretanto, encontrou-se hifas hialinas septadas no material analisado. Diante da possibilidade de infecção fúngica, suspendeu o uso do corticóide tópico e introduziu-se itraconazol via oral por 21 dias, pimaricina tópica associado a tobramicina oftalmológica 4 vezes ao dia e trometamol cetorolaco oftalmológico 3 vezes ao dia em OE.

Em nova consulta 7 dias após início do tratamento antifúngico, paciente relatou melhora parcial dos sintomas, mas BIO de OE ainda com alterações conjuntivais e infiltrados perilimbares. Foi optado por manter o tratamento até completar 21 dias (Figura 1).

Figura 1 - Biomicroscopia de olho esquerdo apresentando hiperemia e lesão ulcerativa em região da conjuntiva e episclera superior.

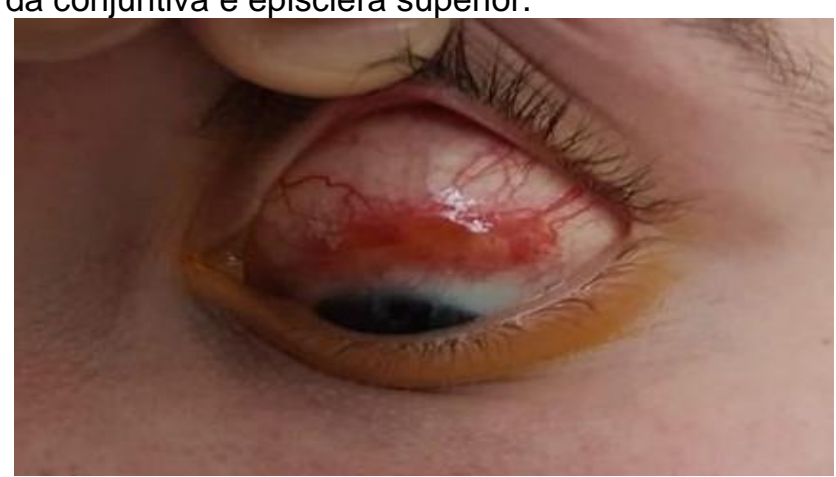

Fonte: Tavares ML, et al., 2021. 
Na terceira reavaliação, após início de antifúngico, paciente referia persistência dos sinais e sintomas, com BIO de OE evidenciando lesão conjuntival superior sem melhora, alguns pontos hemorrágicos e ampliação das margens comprometidas (Figura 2). Infiltrados perilimbares persistiam e foram observadas áreas de afinamentos corneanos periféricos na região superior (Figura 2).

Diante da piora do quadro, foi realizada nova biópsia que revelou a persistência de infiltrado inflamatório crônico, porém, desta vez, com ausência de hifas. Devido à condição do paciente de portador de artrite psoriásica, foi suscitada a hipótese de reação autoimune e o uso de corticóide sistêmico na tentativa de debelar o processo inflamatório ocular. Assim, foram solicitados exames de rastreio para que se pudesse iniciar o tratamento de forma segura.

Figura 2 - Olho esquerdo apresentando piora da hiperemia e lesão ulcerativa em conjuntiva e episclera superior.

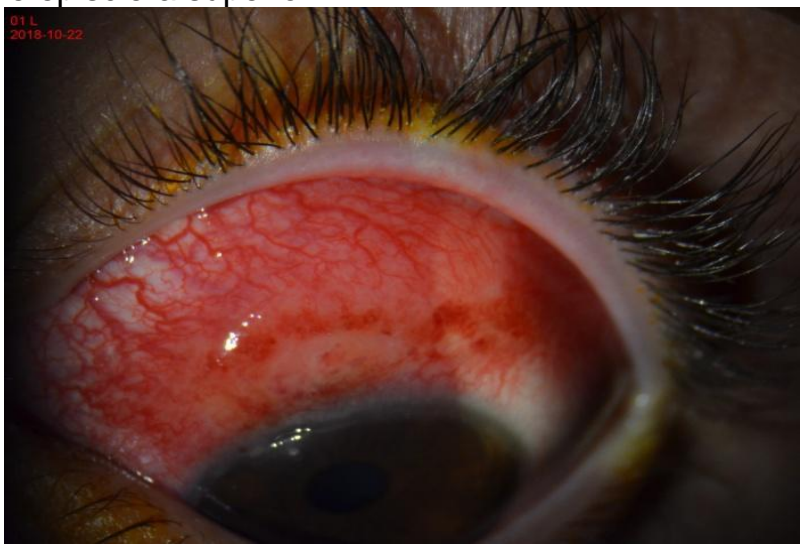

Fonte: Tavares ML, et al., 2021.

Exames de rastreio de doenças infecciosas todos negativos, entretanto foi percebida alteração em teste tuberculínico, com Purified Protein Derivative(PPD) de $21 \mathrm{~mm}$. Na evidência de teste reativo e lesão ocular suspeita, concluiu-se diagnóstico presuntivo de episclerite tuberculosa. Prescrito tratamento com rifampicina $150 \mathrm{mg}$ + isoniazida $75 \mathrm{mg}$ + pirazinamida $400 \mathrm{mg}$ + etambutol $275 \mathrm{mg}$ combinados 4 comprimidos via oral uma vez ao dia por 2 meses. Rifampicina $150 \mathrm{mg}+$ isoniazida $75 \mathrm{mg}$ via oral uma vez ao dia por 4 meses. Complementação com tacrolimus 0,05\% colírio (imunossupressor tópico) 2 vezes ao dia por 4 meses.

Em consulta de reavaliação do quadro, foi relatada melhora dos sintomas visuais já no primeiro mês de tratamento. Após 2 meses de tratamento, paciente retornou apresentando melhora significativa da lesão, com hiperemia em resolução (Figura 3). Mantida conduta até término do tratamento RIPE. A lesão ocular teve regressão completa (Figura 4), apenas com pequeno afinamento perilimbar.

Figura 3 - Olho esquerdo apresentando sinal de melhora com o início do tratamento RIPE.

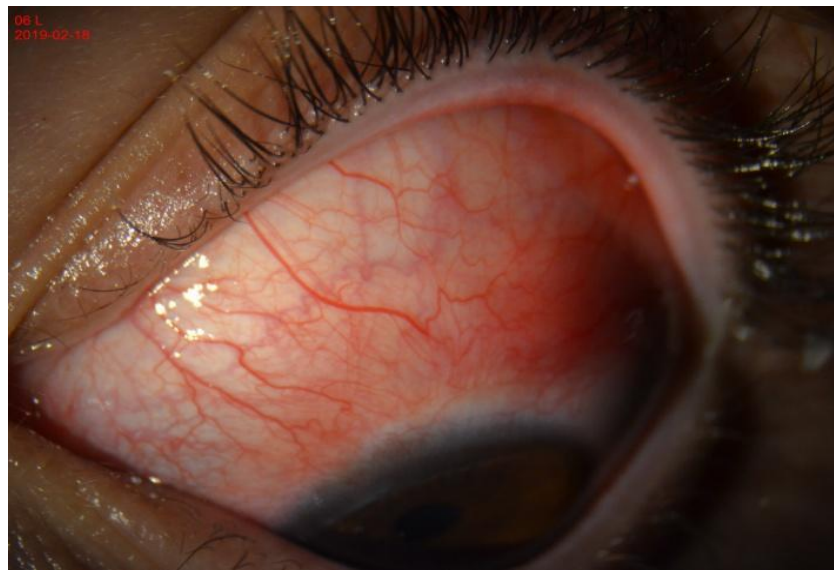

Fonte: Tavares ML, et al., 2021. 


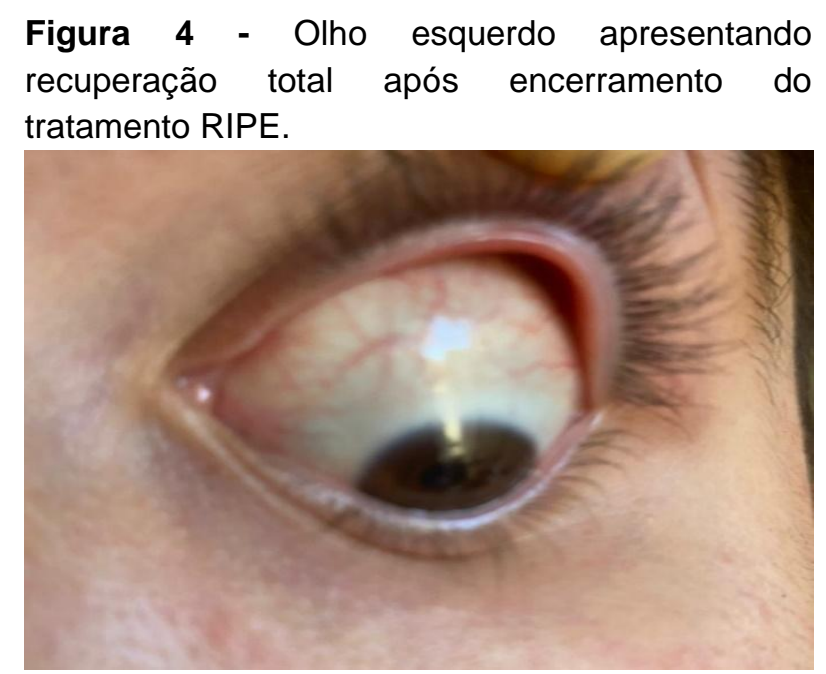

Fonte: Tavares ML, et al., 2021.

\section{DISCUSSÃO}

O presente relato de caso pretende alertar os profissionais de saúde quanto a este possível diagnóstico e estimular a investigação diante de um caso suspeito, já que é estimado que 50 milhões de brasileiros sejam infectados pela tuberculose. Dessa forma poder alertar aos profissionais de saúde sobre as diversas formas de manifestação extrapulmonar que a tuberculose pode vir a desenvolver sem qualquer quadro pulmonar prévio, a exemplo da manifestação ocular; e que muitas das vezes passam despercebidas ao olhar clínico, seja pela falta de experiência teórica, pela escassez de relatos como este, e também pela pouca literatura produzida até o momento, como pela raridade de casos que permitem pouca experiência prática para identificação clínica e também para o manejo terapêutico (BRASIL, 2019; BRASIL, 2021; LAIOS K, et al., 2017).

Outro ponto a ser destacado é que o paciente do relato em caso é portador de espondilite psoriásica, e isso leva a refletir sobre as interações entre a resposta autoimune e a relação com o padrão de resposta ao agente infeccioso, uma vez que o padrão de mecanismo de lesão de tuberculose ocular é por reação de hipersensibilidade do tipo IV (BACK BFC, et al., 2020; CAMPOS WR, et al., 2021). Essa teoria se reforça ainda mais pela boa resposta das lesões oftálmicas ao uso concomitante de imunossupressores no tratamento da tuberculose ocular (KOLLER K, et al., 2019; SOUISSI S, et al., 2017).

Assim se faz importante que mais estudos que façam essa correlação entre a resposta imunológica ao bacilo de Koch e a sua influência sobre doenças de caráter reumatológico sobre os diversos tecidos, especialmente para o trato ocular que até o presente momento apresenta pouca literatura específica. A partir disso possibilitar uma melhor e mais eficiente metodologia diagnóstica, e que possibilitem o desenvolvimento de novas estratégicas terapêuticas a partir do estudo de tal correlação, assim como já se pode perceber em casos que utilizam o tratamento complementar de imunomoduladores para a remissão de lesões extensas e que apresentam maior risco de sequelas ao paciente (CÂMARA SDN, et al., 2019; CAMPOS WR, et al., 2021)

A Tuberculose (TB) é uma patologia infectocontagiosa, causada pelo Mycobacterium tuberculosis, popularmente conhecida como bacilo de Koch. O envolvimento extrapulmonar que inclui manifestações do trato gastrointestinal, trato gênito-urinário, sistema cardiovascular, pele, sistema nervoso central e olhos, pode ocorrer tanto associado a comprometimento pulmonar quanto isoladamente, sem evidência laboratorial ou clínica de infecção pulmonar (CÂMARA SDN, et al., 2019; CAMPOS WR, et al., 2021).

O aparelho ocular é formado por três túnicas: externa (córnea e esclera), intermediária ou uveal (íris, corpo ciliar e coroide) e interna (retina). O mecanismo de envolvimento ocular e dos anexos na tuberculose intraocular inclui invasão dos bacilos ou doença por alterações imunológicas. Esta última se manifesta como 
uma reação de hipersensibilidade que desencadeiam processos inflamatórios, onde estes são responsáveis pelo surgimento de novas manifestações como: doença flictenular, esclerite, ceratite intersticial, uveíte granulomatosa, ou também um quadro de vasculite retiniana.

Conforme alguns estudos o local mais acometido do bulbo ocular na tuberculose intraocular é a úvea, seguido pela retina, ocupando o segundo local mais afetado. Entretanto, qualquer tecido pode ser acometido, como deste relato que foi a episclera do paciente (BANSAL, et al., 2017; CAMPOS WR, et al., 2021).

A tuberculose ocular não é um diagnóstico comum, sendo normalmente baseada no tripé: história clínica, que no caso do paciente do presente relato a queixa principal era irritação em olho esquerdo com saída de secreção matinal, além de achados intraoculares e evidências laboratoriais do Mycobacterium tuberculosis (CÂMARA SDN, 2019; AMARAL GR, et al., 2020; OLIVEIRA SBVD, et al., 2018).

Foram necessárias várias avaliações, duas biópsias, testes terapêuticos e um exame de PPD para se chegar ao diagnóstico. Isto evidencia a dificuldade diagnóstica da tuberculose ocular, tanto por ser rara e que portanto os profissionais de saúde não a colocam como prioridade de diagnóstico diferencial, quanto pela limitação dos exames, já que no caso do nosso paciente as duas biópsias não sugeriram a presença do Mycobacterium tuberculosis, apenas as reações imunológicas com presença de infiltrado crônico e o PPD fortemente reator foram significativos para a hipótese diagnóstica (CAMPOS WR, et al., 2021; AMARAL GR, et al., 2020; KOLLER K, et al., 2019; LAIOS K, et al., 2017; ZAMORA YF, et al., 2019).

O tratamento que é preconizado para a tuberculose ocular é semelhante ao que é receitado para o manejo da tuberculose pulmonar, e de forma geral, a primeira fase desse tratamento consiste no esquema RIPE: Rifampicina, Isoniazida, Pirazinamida e Etambutol, por 2 meses; já na segunda fase do tratamento , faz-se uso de Rifampicina e Isoniazida, por quatro meses; assim como foi realizado o esquema utilizado pelo paciente relatado (BACK BFC, et al., 2020; CAMPOS WR, et al., 2021; AMARAL GR, et al., 2020; KOLLER K, et al., 2019).

Na presença de refratariedade à terapia, deve-se pensar em multirresistência, precisando de avaliação em conjunto com oftalmologistas e especialistas em doenças infecciosas (CAMPOS WR, et al., 2021; NEUHOUSER AJ e SALLAM A, 2020).

O uso de glicocorticóides e imunomoduladores não possuem um fundamento científico fundamentado e comprovado em grande escala para o tratamento de tuberculose ocular, porém em alguns casos é usado, mesmo que de forma bastante criteriosa, por existir a possibilidade de ação anti inflamatória, ajudando a reduzir a progressão de danos, principalmente na tuberculose que acomete a retina (BACK BFC, et al., 2020; CAMPOS WR, et al., 2021; AMARAL GR, et al., 2020; KOLLER K, et al., 2019; SOUISSI S, et al., 2017).

Importante ressaltar que deve ser usado em terapia combinada com o esquema RIPE, devido a possibilidade de reativação da TB latente no olho, devido a imunossupressão, caso seja usado em altas doses. O Tacrolimus foi prescrito por esse princípio anti-inflamatório, e de uso tópico a fim de limitar a ação do fármaco a lesão ocular (BACK BFC, et al., 2020; BRASIL, 2019; CAMPOS WR, et al., 2021).

A resposta adequada e satisfatória ao tratamento estabelecido, reforça a concordância com o diagnóstico presumido de tuberculose ocular, como no caso relatado, que aos dois meses após o início do tratamento, já apresentou resultados bastante significativos (BACK BFC, et al., 2020; BRASIL, 2019; CAMPOS WR, et al., 2021). É necessário que a equipe de oftalmologistas fique sempre em contato com os médicos infectologistas, para que se possa auxiliar no manejo das medicações, uma vez que existe um risco elevado de efeitos colaterais pelo etambutol e da rifanpicina, além dos glicocorticoides sistêmicos, quando administrados (BACK BFC, et al., 2020; BRASIL, 2019; CAMPOS WR, et al., 2021). E também, no caso em questão, a fim de prevenir alguma complicação que poderia desenvolver em associação a patologia autoimune do paciente (espondilite psoriásica).

Portanto, através desse relato e dos estudos que o serviram de base teórica, faz-se perceptível a necessidade que os métodos e protocolos de diagnose sejam atualizados, a fim de que se tornem mais 
eficazes e acurados, visto que em muitas das vezes é possível estabelecer a hipótese diagnóstica sem que haja necessariamente a presença do Mycobacterium tuberculosis à amostra da lesão, como foi o presente relato, bastando apenas a suspeita clínica associada a algum teste de triagem para que se possa estabelecer uma prova terapêutica. Da mesma forma, se faz importante que os protocolos de tratamentos de tuberculose de formas extrapulmonares, sejam difundidos entre os profissionais de saúde, visto que em sua maioria tiveram contato prático ou teórico apenas com casos clássicos de TB; evitando assim que isso possa gerar um certo receio em prescrever o tratamento adequado ao paciente, permitindo uma maior celeridade no processo de cura e evitar a progressão da lesão.

\section{REFERÊNCIAS}

1. AMARAL GR, et al. Tuberculose e suas repercussões oftalmológicas: uma revisão de literatura. Brazilian Journal of Development, 2020; 6(6): 41656-41668.

2. BACK BFC, et al. Tuberculose extrapulmonar ocular: um relato de caso. Revista Médica de Minas Gerais, 2020; 30(6): 23.

3. BANSAL R, et al. Imaging in tuberculosis-associated uveitis. Indian journal of ophthalmology, 2017; 65(4): 264.

4. BRASIL. Manual de Recomendações para o Controle da Tuberculose no Brasil. 2ª ed. Ministério da Saúde, 2019. Disponível em:

https://bvsms.saude.gov.br/bvs/publicacoes/manual_recomendacoes_controle_tuberculose_brasil_2_ed.pdf. Acessado em: 30 de junho de 2021.

5. BRASIL, Ministério da Saúde. Casos confirmados notificados no Sistema de Informação de Agravos de Notificação (SINAN). Disponível em: https://portalsinan.saude.gov.br/. Acessado em: 30 de junho de 2021.

6. CAMPOS WR, et al. Tuberculose intraocular. Revista Brasileira de Oftalmologia, 2011; 70(6): 437-451.

7. CÂMARA SDN, et al. Anterior uveitis as a manifestation of ocular tuberculosis. Revista Brasileira de Oftalmologia, 2019; 78(3): 195-198.

8. DOSHI RR, et al. The spectrum of postoperative scleral necrosis. Survey of ophthalmology, 2013; 58(6): 620-633.

9. FÉNOLLAND JR, et al. Syphilitic scleritis. Ocular immunology and inflammation, 2016; 24(1): 93-95.

10. FERREIRA REBELO PB, et al. Posterior scleritis. Revista Brasileira de Oftalmologia, 2019; 78(2).

11. GUPTA V, et al. Clinics of ocular tuberculosis. Ocular immunology and inflammation, 2015; 23(1): 14-24.

12. KOLLER K, et al. Aspectos clínicos e tratamento de pacientes com tuberculose ocular presumida em centro de referência de São Paulo, Brasil. Estudo retrospectivo. Revista Brasileira de Oftalmologia, 2019; 78(6): 384-388.

13. LAIOS K, et al. The study of ocular tuberculosis during the 19th and early 20th century. Infez Med, 2017; $1(25): 2$.

14. LOUREIRO $\mathrm{M}$, et al. Nodular scleritis associated with herpes zoster virus: an infectious and immune-mediated process. Case reports in ophthalmological medicine, 2016.

15. MARTINEZ AAG, et al. Evolução da esclerite nodular com biomicroscopia ultrassônica: relato de caso. Arquivos Brasileiros de Oftalmologia, 2013; 76(6): 374-376.

16. NEUHOUSER AJ, SALLAM A. Ocular Tuberculosis. StatPearls, 2020.

17. OLIVEIRA SBVD, et al. The impact of ocular tuberculosis on vision after two months of intensive therapy. Brazilian Journal of Infectious Diseases, 2018; 22: 159-165.

18. REBELLO AC, et al. Granuloma macular por tuberculose sem manifestação pulmonar. Revista Brasileira de Oftalmologia, 2015; 74(4): 251-253.

19. REDDY JC, et al. Risk factors and clinical outcomes of bacterial and fungal scleritis at a tertiary eye care hospital. Middle East African journal of ophthalmology, 2015; 22(2): 203.

20. SHAKARCHI FI. Ocular tuberculosis: current perspectives. Clinical ophthalmology, 2015; 9: 2223.

21. SOUISSI S, et al. Steroid treatment in ocular tuberculosis: A double-edged sword? Journal Français D`Ophtalmologie, 2017; 40(2):126-132.

22. ZAMORA YF, et al. Current Practices in Ocular Tuberculosis: A Survey of Brazilian Specialists. Ocular immunology and inflammation, 2019. 